\title{
Intermittent 96 Hour Auricular Electro Acupuncture Using Microcurrent Stimulation for Symptoms of Hot Flashes: Effective Autonomic Modulation?
}

\begin{abstract}
Described here is a new treatment approach for hot flashes with Auricular Electroacupuncture (AEA) using an intermittent 96 hour micro current treatment protocol. This has been tested in a group of men with advanced prostate cancer suffering from vasomotor symptoms (hot flashes) induced by androgen depravation therapy. All subjects were monitored with repeated heart rate variability (HRV) measurements over a six week protocol. The results of the AEA intervention show excellent reduction of hot flash symptoms, along with improved quality of life and sleep. Reviewed here are aspects of this treatment protocol that support continued research with AEA for this model disease that has pathophysiologic underpinnings of dysfunctional autonomic nervous system activity. The improvement of clinical symptoms with AEA intervention may represent autonomic modulation resulting from a balanced sympathetic and parasympathetic activity produced by prolonged auricular vagal nerve stimulation.
\end{abstract}

Keywords: Vasomotor symptoms; Hot flashes; Autonomic nervous system; Heart rate variability

Short Communication
Volume 9 Issue 3 - 2017
Tyvin Rich ${ }^{1 *}$, Tzvi Milshtein ${ }^{2}$ and Thomas
Corbin $^{3}$
${ }^{1}{ }^{2}$ Hampton University Proton Therapy Institute, USA
${ }^{2}$ CM Wellness Clinic, USA
${ }^{3}$ West Palm Beach Veterans Hospital, USA
*Corresponding author: Tyvin Rich, MD FACR, Hampton
University Proton Therapy Institute, 40 Enterprise Parkway,
Hampton, VA 23666, USA, Tel: 757 251 6819;
Email: tyvinr@gmail.com
Received: November 11, 2017 | Published: November 20,
2017

Abbreviations: AEA: Auricular Electroacupuncture; ADT: Andogen Deprovation Therapy; HRV: Heart Rate Variability; LF: Low Frequency; HF: High Frequency

\section{Introduction}

Where do hot flashes (an autonomic disorder) come from?

In a recent publication we describe the major subjective improvement of hot flashes and sleep disruption caused by hormone blockade in prostate cancer patients treated with Auricular Electro Acupuncture (AEA) [1]. Hot flashes are troublesome vasomotor symptoms caused by gender hormone withdrawal that can be caused by androgen depravation therapy (ADT) [2], in breast cancer survivors after chemo/hormonal therapy [3], or naturally in menopausal women [4]. Several reports show that hot flashes are identical in both sexes $[5,6]$ making their study a model disease condition for the evaluation of medical and non-pharmacologic interventions. Along with the vasomotor events, a constellation of other debilitating symptoms occur including fatigue, weight gain, anxiety and depression, loss of libido, and changes of patterns or disruption of sleep. The current understanding of the patho-physiology of vasomotor symptoms is well reviewed by publications by Freedman and others $[7,8]$. The broad overview of the patho physiology starts first with a required transition from normal to reduced gender hormone levels. This disrupts hypothalamic monitoring of the thermoneutral zone where small increases in body temperature results in a "confused" top/down signal to the ANS network [9].
Sympathetic activation occurs as confirmed with altered galvanic skin resistance measurements indicating excitation of sweat glands by sympathetic cholinergic fibers. Biochemical data also show sympathetic activation based on elevations of the plasma norepinephrine metabolite, 3-methoxy-4-hydroxyphenylglycol increased in of patients with hot flashes, and pharmacologic interventions with Yohimbine, a $\alpha 2$-adrenergic antagonist, and with clonidine (a $\alpha 2$-adrenergic agonist) have been used for the treatment of hot flashes [9]. Other corroborating evidence supporting sympathetic activation with a hot flash is based on heart rate variability (HRV) data showing elevated low frequency (LF) HRV during a 5-min block at the onset of a nocturnal physiologic hot flash compared to a 5-min block after a hot flash, regardless of sleep or wake state [10]. Another HRV study of hot flashes patients revealed that there is also high frequency signal loss in perimenopausal subjects during non-REM sleep indicating that both the sympathetic and parasympathetic branches of the ANS are altered during a hot flash [11]. These data suggest hot flashes and sleep dysfunction in particular, could be hypothesized to be a disturbance of the autonomic nervous system (ANS). Supporting data for this point are from a recent fMRI study showing the temporal sequence of vasomotor attacks are first detected in ANS centers in the brain stem. The early pre-clinical onset of brain stem activation prior to higher interoceptive detection (in the insular cortex and prefrontal lobe sites) of a hot flash suggests the brains stem is a critical functional center whereas the higher brain centers are associated with the phenomenological correlates of hot flashes [12]. Targeting of the ANS thus makes sense as a therapeutic intervention for hot flashes. 


\section{How effective are standard medical interventions or alternative treatments for hot flashes?}

Treatment recommendations for medical and or complementary interventions for hot flashes are well described in a robust medical literature and the reader can find multiple internet websites with a plethora of information. One agreed upon but controversial treatment, hormone replacement, is usually reserved for very symptomatic female subjects. However, reservations about hormone replacement therapy exist because of known risks of cancer induction that make the usual recommendation in this situation for the lowest dose over the shortest period to ameliorate symptoms. These agents and most other medical interventions have not been very successful and in some cases carry not only the additional burden of cancer, but cardiovascular events (clotting and stroke), and aggravation of heart disease. Other treatments that have been used with limited success are estrogen receptor modulators (in females), selective serotonin reuptake inhibitors, Gabapentin, Clonidine, vitamin E, physical exercise, deep breathing, relaxation techniques, and lowering ambient room temperature. Intervention trials show there is a 20 to $30 \%$ placebo effect that can confound the results and must be recognized when testing new interventions [13].

The unsatisfactory results of medical interventions has helped usher in exploration of alternative approaches like body acupuncture for the management of vasomotor symptoms. The practice of body acupuncture for these patients has been extensively reviewed in a Cochrane analysis [14] where in 16 randomized controlled trials ( $\mathrm{n}=1155$ women) found no evidence that real acupuncture compared to sham made a difference. More recently, other well- powered prospective randomized trials confirmed that body acupuncture was no better than control intervention in women with menopausal HFs $[15,16]$. Because of the relatively poor success overall with body acupuncture for this disorder and emerging knowledge of the utility of AEA for modulation of the autonomic nervous system [17], we chose to evaluate the Neurova device that can deliver an intermittent micro current stimulation to ear points over 96 hours. The purpose of our pilot trial was to determine the effect of AEA on hot flashes, quality of life (QoL), and sleep, and monitoring the continuous treatment with HRV.

\section{Intermittent 96 hour Auricular Electroacupuncture: a new treatment paradigm for hot flashes?}

We have shown that the use of an intermittent 96 hour treatment with AEA appears to be very effective for a small cohort of men with hot flashes [1]. In 10 subjects there were highly significant improvements of the quality of life, sleep, and hot flash frequency and duration over the six weeks of the protocol study. Although there is always the concern of a placebo effect with new interventions for hot flashes, we showed that all patients have improvement in their objective HRV LF/HF ratios over the course of the protocol intervention. Furthermore, we interpret the change in LF/HF ratios to reflect improved sympathovagal balance produced by neuro modulation. Neuroanatomic correlates of the
AEA points used in our protocol are supported by imaging data showing that Shen Men, Point Zero, and Autonomic, activate classic vagal projections in the brain stem [18]. Further support comes from a recent study of human volunteers indicating that auricular transcutaneous vagal nerve stimulation also produces sympathetic deactivation (measured by microneurography) [19]. These data are consistent with our hypothesis that ANS modulation by intermittent 96 hour microcurrent AEA occurs by targeting parasympathetic stimulation that can also modify sympathetic activity.

\section{AEA with HRV Monitoring}

The tools used in our study are two devices designed to deliver intermittent AEA for up to four days. The Neurova is an FDA approved device shown in Figure 1. It can be used for patients with pain, addiction, depression and anxiety, and inflammatory conditions by choosing the appropriate ear points. The other device we employed is the BlueCardio (Figure 2), a heart rate monitor designed to detect a participant's heart rate for 300 second epochs. This measurement can be used repeatedly over the day by the patient allowing for convenient data collection before, during, and after AEA treatment. The patient is asked to sit comfortably and to breath normally during each data acquisition session. The BlueCardio is Bluetooth capable and can be securely linked to an application loaded onto the subject's smart phone or a wi fi enabled tablet that sends the heart data for time and frequency domain analysis by the Kubios software by a HIPPA compliant internet server. These two devices allow mobile data collection in the private setting of the patient's environment without stress or lifestyle disruption. These devices can be combined with validated patient questionnaires to objectively score the symptoms being treated over the course of treatment and to possibly monitor objectively the trajectory of the treatment effect. In our ongoing protocol, we combine BlueCardio measurements with AEA intervention plus minimally invasive detection of other objective parameters associated with hot flashes like galvanic skin resistance and core body temperature measurements.
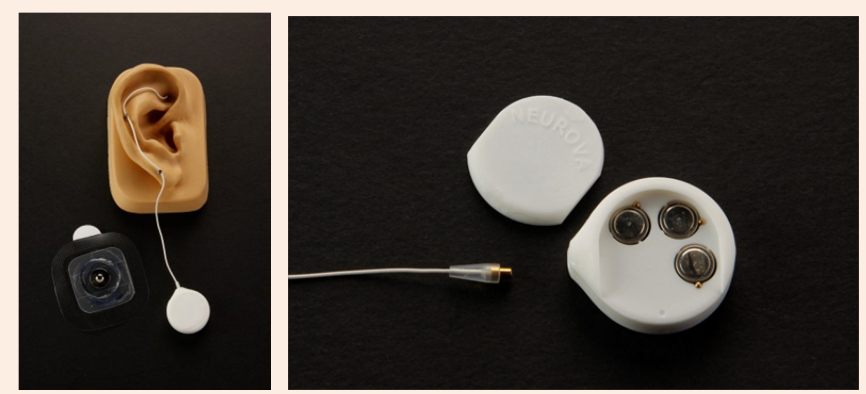

Figure 1: The Neurova device consists of a wire with three sterile needles that are inserted into selected ear points that is connected to a plastic unit housing the power source and programmed chip (above) that delivers the AEA treatment. A gel sticky pad is placed behind the ear to complete the circuit. The patient can disconnect the unit themselves, bathe, and reconnect each day using a new gel pad. 


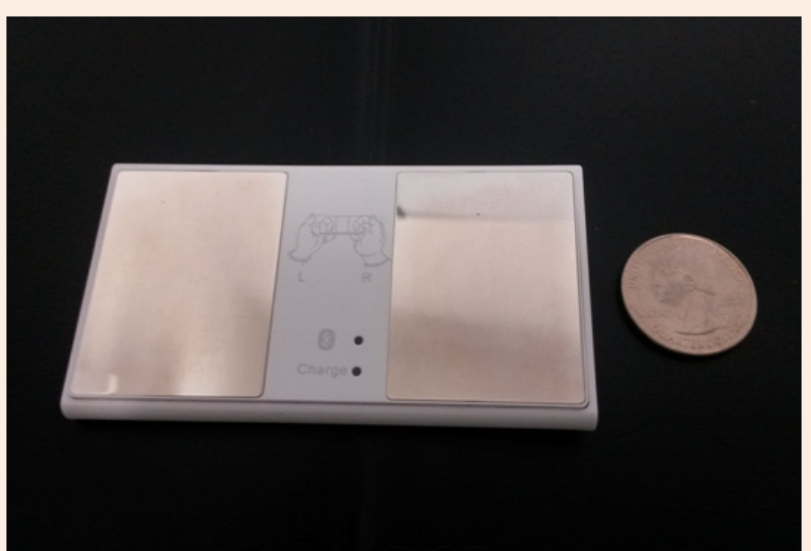

Figure 2: The BlueCardio device used to collect heart rate. The 300 second data collection is then sent to a smart phone application where it can be uploaded to an internet server for data analysis.

\section{Summary}

Our treatment protocol using intermittent 96 hour AEA for hot flashes has demonstrated a potential clinical utility for men with advanced prostate cancer treated with ADT. The results appear better than traditional body acupuncture interventions, possibly on the basis of a longer duration of treatment compared to typical weekly body acupuncture sessions. The AEA protocol uses stimulation of neural projections by the auricular branch of the vagus nerve to known vagal afferent centers that, in turn, may modulate the sympathetic side of the ANS. These findings are consistent with an emerging medical view that vagal nerve stimulation shows promise for the non pharmacologic management of conditions like drug resistant epilepsy, inflammation, depression, sleep disorders, PTSD, anxiety, and treatment resistant depression. Further trials with intermittent 96 hour AEA are justified.

\section{Conclusion}

AEA using an intermittent 96 hour protocol has been found to improve vasomotor symptoms in a small pilot trial in men treated with ADT. The AEA treatment can be monitored with HRV and managed by the patient at home while the data can be monitored by the physician remotely, using a web compliant and convenient device to repeatedly assess the patient's ANS status throughout the day. Our preliminary findings are encouraging to warrant continued evaluation of this treatment paradigm for both men and women with hot flashes and possibly other medical conditions with a component of autonomic dysfunction.

\section{Acknowledgement}

The authors wish to thank the support of the medical, nursing, and technical staff of the Hampton University Proton Therapy Institute, Hampton, VA enabling our prospective protocol for the evaluation of AEA in men with advanced prostate cancer while undergoing curative proton irradiation.

\section{Conflicts}

The authors have financial conflicts to disclose.

\section{References}

1. Rich TA, Porter GW, Ricks-Santi L, Milshtein T, Corbin T (2017) Intermittent 96 hour auricular electroacupuncture for hot flashes in prostate cancer: a pilot study. Med Acupunct 29(5): 313-321.

2. Shahinian VB, Kuo YF, Freeman JL, Orihuela E, Goodwin JS (2005) Increasing use of gonadotropin-releasing hormone agonists for the treatment of localized prostate carcinoma. Cancer 103(8): 16151624.

3. Loprinzi CL, Barton DL, Rhodes D (2001) Management of hot flashes in breast-cancer survivors. Lancet Oncol 2(4): 199-204.

4. Kronenberg F (1990) Hot flashes: epidemiology and physiology. Ann N Y Acad Sci 592: 52-86.

5. Engstrom CA, Kasper CE (2007) Physiology and Endocrinology of hot flashes in Prostate Cancer. American J Mens Health 1(1): 8-17.

6. Jones JM, Kuhli M, Loprinzi CL (2012) Androgen deprivation therapy -asspciated hot flashes. Asian J of Andrology 14: 193-197.

7. Thurston RC, Christie IC, Matthews KA (2012) Hot flashes and cardiac vagal control during women's daily lives. Menopause 19(4): 406-412.

8. Thurston RC, Santoro N, Matthews KA (2013) Are vasomotor symptoms associated with sleep characteristcs among symptomatic midlife women? Comparisons of self-report and objective measures. Menopause 19(7): 742-748.

9. Freedman RR (2001) Physiology of hot flashes. Am J Hum Biol 13(4): 453-464.

10. Freedman RR, Kruger ML, Wasson SL (2011) Heart rate variability in menopausal hot flashes during sleep. Menopause 18(8): 897-900.

11. de Zambotti M, Colrain IM, Sassoon SA, Nicholas CL, Trinder J, et al. (2013) Vagal withdrawal during hot flashes occurring in undisturbed sleep: Hot flashes and autonomic activity. Menopause 20(11): 11471153.

12. Diwadkar VA, Murphy ER, Freedman RR (2014) Temporal sequencing of brain activations during naturlly occuring thermoregulatory events. Cereb Cortex 24(11): 5006-5013.

13. Sloan JA, Loprinzi CL, Novotny PJ, Barton DL, Lavasseur BI, et al. (2001) Methodologic lessons learned from hot flash studies. J Clin Oncol 19(23): 4280-4290.

14. Dodin S, Blanchet C, Marc I, Ernst E, Wu T, et al. (2001) Acupuncture for menopausal hot flushes; Cochrane Database of Systematic Reviews Issue 7.

15. Vincent A, Barton DL, Mandrekar JN, Cha SS, Zais T, et al. (2007) Acupuncture for hot flashes: a randomized, sham-controlled clinical study. Menopause 14(1): 45-52.

16. Ee C, Xue C, Chondros P, Myers SP, French SD, et al. (2016) Acupuncture for Menopausal Hot Flashes: A Randomized Trial. Ann Intern Med 164(3): 146-154.

17. He W, Wang X, Shi H, (2012) Auricular acupuncture and vagal regulation. Evidence Based Complemtary and Alternative Medicine 786839.

18. Frangos E, Ellrich J, Komisaruk BR (2015) Non-invasive access to the vagus nerve central projections via electrical stimulation of the external ear: fMRI evidence in humans. Brain stimul 8(3): 624-636.

19. Clancy JA, Mary DA, Witte KK, Greenwood JP, Deuchars SA, et al. (2014) Non-invasive vagus nerve stimulation in healthy human volunteers reduces sympathetic nerve activity. Brain Stimul 7(6): 871-877. 